SCIENTIFIC REPORT

\title{
Does the site of filtration influence the medium to long term intraocular pressure control following microtrabeculectomy in low risk eyes?
}

\author{
A K Negi, A W Kiel, S A Vernon
}

Br J Ophthalmol 2004;88:1008-1011. doi: 10.1136/bjo.2003.028811

Aims: To evaluate the influence of varying surgical site on the medium to long term intraocular pressure (IOP) control in patients undergoing unenhanced small flap trabeculectomy (microtrabeculectomy) in eyes at low risk of failure.

Methods: A retrospective non-concurrent analysis was performed on two cohorts of patients who underwent unenhanced microtrabeculectomy at different sites by a single surgeon (SAV). The first cohort of eyes was part of a trial to study the astigmatic effect of microtrabeculectomy (results published elsewhere) and all had flaps centred at the 12 o'clock meridian (superior flaps). The second cohort consisted of eyes with flaps created on either side of the 12 o'clock - that is, superonasal in left eyes and superotemporal in right eyes. All case notes were reviewed for the preoperative and presenting IOPs, the number and duration of antiglaucoma medication use preoperatively and, postoperatively, any intraoperative, early, or late postoperative complications. All IOPs measured at 6 months and then yearly intervals were recorded. The baseline characteristics and IOPs at each follow up were compared between the eyes with the superonasal and superotemporal flaps of the non-12 o'clock group against those with superior flaps in the 12 o'clock group up to a maximum of 72 months. Survival was assessed by the site of microtrabeculectomy, with failure considered as any IOP above 22 and $15 \mathrm{~mm} \mathrm{Hg}$ with or without medications.

Results: All patients had a minimum follow up of 12 months and 12/17 patients in the $12 o^{\prime}$ clock group and 17/28 in the non-12 o'clock group completed the full follow up of 72 months. The IOPs at all points in time were lower in the left eyes with superonasal flaps compared to both the superior and the superotemporal groups. This difference was statistically significant between the three groups to the end of 4 years $(p=0.001)$ and remains clinically significant thereafter with the mean last recorded IOPs of 15.9, $12.4(p=0.03)$, and $14.3 \mathrm{~mm} \mathrm{Hg}$ in the superior, superonasal and superotemporal groups respectively, with a smaller mean number of drops in the non-12 o'clock group. Kaplan-Meier curves showed a significantly better outcome for the cutoff IOP of $15 \mathrm{~mm} \mathrm{Hg}$ in the superonasal group $(p=0.003)$ compared with both the other groups.

Conclusion: Eyes with superonasal flaps achieve and maintain lower IOPs when compared with both the superior and superotemporal flaps. The results suggest that, when a low target IOP is desired, the site of surgery in an unenhanced filtering procedure should be superonasally sited.

S ince its original description more than three decades ago trabeculectomy has remained the gold standard of filtration surgery. The basic technique has remained the same; however, various minor modifications altering the shape $^{1}$ and $\operatorname{size}^{23}$ of the superficial flap and sclerostomy, the use of limbal or fornix based conjunctival flaps, ${ }^{4}$ and the method for achieving the deep sclerostomy by trephination ${ }^{5}$ or use of a scleral punch ${ }^{6}$ have been tried.

Conventionally, initial trabeculectomies are performed at the $12 \mathrm{o}^{\prime} \mathrm{clock}^{7}$ limbus because of the ease of access and the protection afforded to the bleb by the upper lid.

Sanders $\mathrm{et}^{\mathrm{a} l^{8}}$ reported lower intraocular pressures (IOPs) over a short term of 18 months with standard sized trabeculectomies performed superonasally. One of the authors (SAV) has previously published his technique of microtrabeculectomy and its successful medium to long term outcome in low risk patients. ${ }^{3}$ The initial report ${ }^{3}$ also found that a superonasal flaps achieved lower IOPs during the first year following surgery.

This study examines the hypothesis that site of surgery affects long term IOP control in trabeculectomy in low risk patients.

\section{PATIENTS AND METHODS}

During the period between May 1995 and May 1996 a consecutive cohort of 17 patients underwent microtrabeculectomy intentionally performed superiorly at 12 o'clock to investigate the astigmatic effect of small flap microtrabeculectomies. ${ }^{10}$ We retrospectively compared the IOP control in this group of patients with a control cohort of 28 consecutive eyes from 28 patients who underwent unenhanced microtrabeculectomies, also performed by the same surgeon (SAV), during an earlier period between January 1991 and January 1995, using an identical surgical technique but with operating sites on either side of the vertical meridian (non-12 $o^{\prime}$ clock cohort). These were uniformly superonasal in the left and superotemporal in the right eyes (such sitings have been routine practice by the surgeon who is right handed). "Small flap" trabeculectomy (microtrabeculectomy) is a much scaled down modification of the "full size" procedure. The full surgical technique and its potential advantages have been described elsewhere. ${ }^{39}$

Only the first eyes of white European patients were included. All case notes were reviewed to collect data including the baseline characteristics, any surgical complications, and postoperative interventions including suturelysis or chamber reformations. Any additional surgical procedures in the follow up period were noted, including needling for encysted blebs, repeat filtration procedure, or need for cataract surgery. All IOPs, as measured by Goldmann applanation tonometry, were recorded at follow up visits at 3 and 6 months and then yearly until the last recorded follow up.

Abbreviations: IOP, intraocular pressure; POAG, primary open angle glaucoma 
Table 1 Baseline characteristics of the patients included in the analysis

\begin{tabular}{|c|c|c|c|c|}
\hline & Superior (12 o'clock) & Superonasal (left eyes) & Superotemporal (right eyes) & p Value* \\
\hline No of eyes & 17 & 13 & 15 & \\
\hline Male: female & $13: 4$ & $7: 6$ & $8: 7$ & \\
\hline Mean age at the time of surgery (SD), (years) & $67.4(10.8)$ & $71.7(10.1)$ & $71(9.9)$ & 0.37 \\
\hline Range & $45-81$ & $51-85$ & $46-83$ & \\
\hline Mean preop IOP (SD) $(\mathrm{mm} \mathrm{Hg})$ & $24.9(5.2)$ & $25.7(3.7)$ & $24.5(5.1)$ & 0.74 \\
\hline Diagnosis to operation (months) & 29.6 & 38.3 & 36.1 & 0.64 \\
\hline Mean follow up (months) & 60.8 & 54.8 & 49.4 & 0.14 \\
\hline Mean no of drops preop (SD) & $1.1(0.33)$ & $1.5(0.63)$ & $1.4(0.63)$ & 0.19 \\
\hline Mean no of drops at last follow up (SD) & $0.71(1.04)$ & $0.23(0.60)$ & $0.20(0.41)$ & 0.36 \\
\hline $\begin{array}{l}\text { Average time to the addition of first glaucoma } \\
\text { medication following surgery (months) }\end{array}$ & 23 & 40.5 & 30.6 & 0.40 \\
\hline $\begin{array}{l}\text { Average IOP at the start of the first glaucoma } \\
\text { medication following surgery (mm Hg) }\end{array}$ & 26 & 21 & 21.3 & 0.44 \\
\hline
\end{tabular}

Baseline characteristics of the three groups, preoperative and postoperative IOPs, number and duration of use of antiglaucoma drops preoperatively, antiglaucoma treatment required during the follow up and number of drops at last index visit, and the mean IOPs at each follow up point were analysed using the non parametric Kruksal-Wallis test. Long term IOP survival was analysed according to the site of operation-that is, superior against superonasal (left eyes) and superotemporal (right eyes), considering IOPs of $>22$ and $>15 \mathrm{~mm} \mathrm{Hg}$ (with or without medications) as failure. Kaplan-Meier survival curves were created and differences analysed with the Mantel-Cox log rank test censoring incomplete data. All statistical analysis was performed and graphs created using "Stat view" software (SAS Institute Inc).

\section{RESULTS}

There were 17 eyes in the 12 o'clock group and 28 in the non12 o'clock group completing the minimum follow up of 12 months, and $12(70.6 \%)$ and $14(50 \%)$ respectively completed the full follow up of 72 months. In the 12 o'clock group, three patients died and two were lost to follow up while in the non-12 o'clock group seven patients were lost to follow up and seven had died during the period.

In the 12 o'clock group two eyes had primary open angle glaucoma (POAG) with narrow but non occluded angles and the remaining $(88 \%)$ had fully open angles, while in the non12 o'clock group 25 eyes (89\%) had POAG, one had pigmentary glaucoma, and two had glaucoma associated with pseudoexfoliation syndrome.

There was no statistically significant difference between the baseline characteristics of the superior, superonasal, and superotemporal groups (table 1). After the first 6 months of follow up, all measured IOPs were statistically significantly lower in the superonasal group compared to both superotemporal and the superior group (table 2). This difference was highly statistically significant for each follow up up to 4 years $(p=0.001)$. The IOPs in the superonasal group continue to remain lower at 5 and 6 years but differences are not statistically significant possibly because of the small number of eyes completing the full follow up. However, the last recorded IOP in the superonasal group (12.4 mm Hg) was statistically significantly lower than the superior group ( $15.9 \mathrm{~mm} \mathrm{Hg}$, p value for Mann-Whitney test $=0.03$ ). Superior flaps showed better survival outcome for both 22 and $15 \mathrm{~mm} \mathrm{Hg}$ cut off (fig $\mathrm{l}$ ).

Eleven $(39.3 \%)$ patients in the non-12 group (five in superonasal and six in superotemoral group) had hyphaema compared to only one in the superior group $(p=0.01)$. One eye in the superotemporal group underwent reformation for a flat anterior chamber and one each in superonasal and
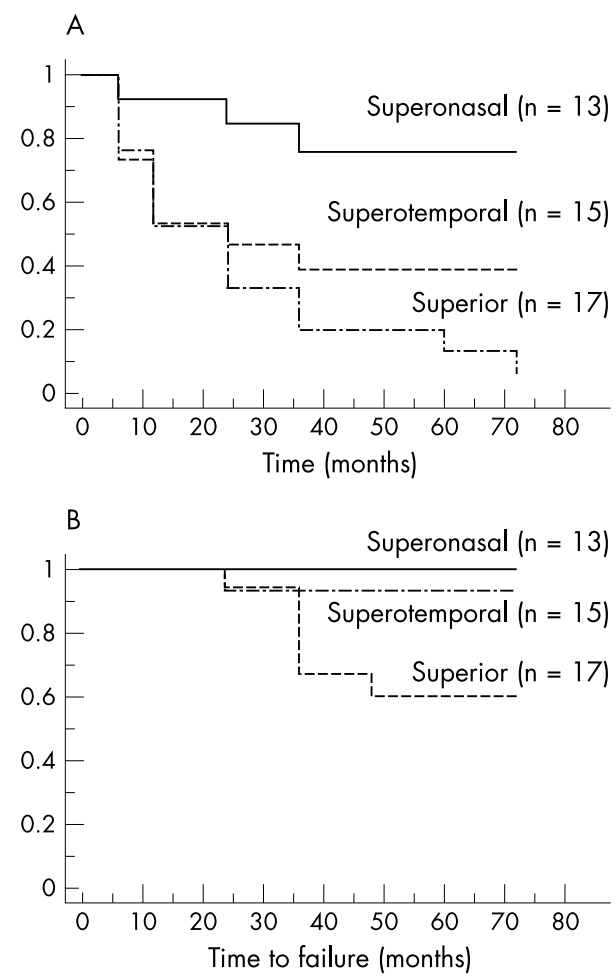

Figure 1 (A) Kaplan-Meier survival curves for any IOP $>15 \mathrm{~mm} \mathrm{Hg}$ with or without treatment considered as failure. The $p$ value on the log rank test was 0.003 . (B) Survival curve for any $I O P>22 \mathrm{~mm} \mathrm{Hg}$ with or without treatment considered as failure. The $\mathrm{p}$ value for the log rank test was 0.008 between the survival for superonasal (left eyes) and superior groups.

superotemporal group had blebitis compared to none in the superior group. Two patients in the superior group and one patient each in the superotemoral and superonasal group required suturelysis. In the superior group both these patients eventually required topical antiglaucoma medications between 10 and 12 months postoperatively. In the superonasal group, the patient requiring suturelysis maintained good IOP control, while the one in the superotemporal group needed antiglaucoma medication. One patient in the superior group required needling for encysted bleb and another underwent repeat microtrabeculectomy with antimetabolite for uncontrolled IOP after 5 years of initial surgery.

Eleven (39\%) eyes in the non-12 o'clock group (five out of 13 in the superonasal group and six out of 15 in the superotemporal group) required cataract surgery compared to 
Table 2 Mean IOPs (number of eyes at each visit) in the superior, superonasal, and superotemporal groups at each point in time during the follow up

\begin{tabular}{|c|c|c|c|c|c|c|c|c|c|}
\hline & \multicolumn{2}{|c|}{ Presenting IOP (n) 6 months } & \multirow{2}{*}{$\begin{array}{l}1 \text { year } \\
15.3(17)\end{array}$} & \multirow{2}{*}{$\begin{array}{l}2 \text { years } \\
16.5(16)\end{array}$} & \multirow{2}{*}{$\begin{array}{l}3 \text { years } \\
17.7(15)\end{array}$} & \multirow{2}{*}{$\begin{array}{l}4 \text { years } \\
17.1(14)\end{array}$} & \multirow{2}{*}{$\begin{array}{l}5 \text { years } \\
16.7(13)\end{array}$} & \multirow{2}{*}{$\begin{array}{l}6 \text { years } \\
16.7(12)\end{array}$} & \multirow{2}{*}{$\begin{array}{l}\text { Last IOP } \\
15.9 \text { (17) }\end{array}$} \\
\hline Superior (12 o'clock) & 30.4 (17) & $12.6(17)$ & & & & & & & \\
\hline Superonasal (left eyes) & $33.0(13)$ & $8.8(13)$ & $9.8(13)$ & $10.9(13)$ & $12.3(12)$ & $10.5(8)$ & $12.9(8)$ & $12.1(7)$ & 12.4 \\
\hline Superotemporal (right eyes) & $32.9\left(14^{*}\right)$ & $12.7(15)$ & $14.5(15)$ & $15.1(15)$ & $14.9(11)$ & $15.9(8)$ & $13.3(7)$ & $13.0(7)$ & 14.3 \\
\hline $\mathrm{p}$ Valuest & 0.18 & 0.02 & 0.003 & 0.008 & 0.01 & 0.001 & 0.15 & 0.10 & 0.07 \\
\hline
\end{tabular}

*Presenting IOP was not recorded for one eye.

†From the Kruksall-Wallis test for unpaired comparisons for more than two groups.

only one $(6 \%)$ in the superior group $(p=0.01)$. The average age at the time of filtering surgery of the patients in the non12 o'clock group, who later required cataract surgery was 75.9 years (SD 5.9) compared to 68.3 years (SD 10.8), in those who did not $(p=0.09)$. The mean duration to the time of cataract surgery from the time of trabeculectomy was 33 months (median 30, range 1-72 months).

\section{DISCUSSION}

Trabeculectomy is currently the most commonly performed operation for the surgical treatment of open angle glaucoma. Small scleral flaps have been shown to provide IOP control similar to that found in conventional sized procedures. ${ }^{239}$

Cairns, at the time of his original description, suggested that a trabeculectomy could be performed anywhere along the limbus. ${ }^{7}$ Sanders $\mathrm{et}_{\mathrm{al}}^{8}$ first reported the effect of varying site on the outcome of trabeculectomy in a prospective trial with 60 patients randomised into three groups. They reported lower IOPs at each follow up in the nasal group, with 30\% patients having IOPs of $<10 \mathrm{~mm} \mathrm{Hg}$ (compared to $10 \%$ in the superior group and none in the temporal group) at the end of 18 months.

Having found similar results to Sanders et $a l^{8}$ in our initial report on microtrabeculectomy ${ }^{3}$ we analysed the IOP lowering effect and survival between the left and right eyes of the non-12 o'clock group over a longer period of time. The eyes with superonasal flaps achieved lower IOPs with fewer antiglaucoma drops at all follow ups and showed better survival with any IOP $>15 \mathrm{~mm} \mathrm{Hg}$ counted as a failure.

More eyes in the non-12 o'clock group (39.3\%) had hyphaema; however, the rates are comparable to those published in the literature of $7 \%$ to $53 \%,{ }^{11}{ }^{12}$ and did not differ significantly between the superonasal and superotemporal sites.

Sanders et $a l^{8}$ reported more bleb related complications in their nasal group with $20 \%$ showing bleb leaks. They also reported a $30 \%$ rate of corneal dellen formation this group compared to $10 \%$ in the temporal and none in the superior group. We had two cases of blebitis in the non-12 o'clock group one each in the superonasal and superotemporal group. The first one was diagnosed at 72 months postoperatively and was associated with bleb leak which was treated with topical antibiotics and autologous blood injection, but eventually required bleb revision resulting in a successful outcome and IOP control without any medications. The second patient had late blebitis treated successfully with topical antibiotics alone. None of our patients required early resuturing for bleb leak and there were no cases of corneal dellen formation. We believe this to be a result of fastidious flap control and conjunctival suturing peroperatively, which is part of the protocol of microtrabeculectomy. Budenz et $a l^{13}$ reported a higher incidence of bleb related dysaesthesia in eyes with superonasal blebs following standard sized trabeculectomies. Being a retrospective study we did not formally check this; however, we are not aware of any significant bleb related problem except the one case of blebitis in the superonasal group.

Interestingly, more eyes in superonasal and superotemporal groups needed cataract surgery in the medium to long term. However, this is in keeping with the incidence of cataract reported after conventional trabeculectomy of between $35 \%$ and $40 \% .{ }^{14}$ The risk of cataract formation is known to be increased in eyes of older patients with excessive postoperative inflammation and those which have suffered flat or shallow anterior chambers. ${ }^{15}$ None of our patients had excessive postoperative inflammation and only one eye in the superonasal group had a flat anterior chamber in the early postoperative period (requiring chamber reformation). This eye eventually underwent lens extraction for early intumescent cataract 1 month after the initial microtrabeculectomy.

The main limitations our study are its retrospective nature and a relatively small number of eyes completing the full follow up. We acknowledge that the success of any treatment should be measured in terms of its effectiveness in preventing disease progression, which in glaucoma means halting or slowing progressive visual field loss. However, the one single factor most consistently reported to be linked to progressive field loss has been the intraocular pressure ${ }^{16}$ and therefore continually low IOP levels can be considered to act as surrogate for field survival in studies with a small number of eyes/patients.

The possible mechanisms for the improved IOP control in nasally sited procedures could be a structural variation in the anatomy of conjunctiva or sclera at different sites or a reduced mechanical compression effect of the upper lid superonasally thereby allowing a different bleb morphology to develop in the early post operative phase. We have noted reduced episcleral tissue density and adhesions between conjunctiva and episclera nasally but have not formally quantified this.

Our findings are only appropriate to non-enhanced filtering procedures with limbus based conjunctival flaps, although no difference in IOP control has been found with fornix based procedures. ${ }^{4}$ Further analysis of the results of antimetabolite enhanced operations (performed in a similar manner concerning the site of operation) will aid our ability to be able to predict IOP levels following filtering surgery.

With lower target IOPs recommended for better long term control of glaucoma, our study would suggest consideration for fashioning the trabeculectomy flaps on either side of the vertical meridian and siting the operation nasally when maximum IOP control is desirable.

\section{Authors' affiliations}

A K Negi, A W Kiel, S A Vernon, Department of Ophthalmology, Eye and ENT Centre, University Hospital, Nottingham, UK

None of the authors has any financial or proprietary interest in any of the products or techniques mentioned in this paper.

Presented in part at the UK and Eire Glaucoma society annual meeting, November 2002. 
Correspondence to: Mr S A Vernon, Eye and ENT Centre, University Hospital, Nottingham NG7 2UH, UK; Stephen.Vernon@mail.qmcuh-tr. trent.nhs.uk

Accepted for publication 15 December 2003

\section{REFERENCES}

1 Krasnov MM. A modified trabeculectomy. Ann Ophthalmol 1974;6:178-82.

2 Starita RJ, Fellman RL, Spaeth GL, et al. Effect of varying the size of scleral flap and corneal block on trabeculectomy. Ophthalmic Surg 1984;15:484-7.

3 Vernon SA, Spencer AF. Intraocular pressure control following microtrabeculectomy. Eye 1995;9:299-303.

4 Schuster R, Krupin T, Kolker AE, et al. Limbus v fornix-based conjunctival flap in trabeculectomy: a long term randomised study. Arch Ophthalmol 1984; 102:361-2.

5 Dellaporto A. Experience with trephanotrabeculectomy. Trans Am Acad Ophthalmol Otolaryngol 1975;79:362-71.

6 Tomey KF, Shamas IV. Single snip trabeculectomy using a specially designed punch. Ophthalmic Surg 1986;17:816-8.

7 Cairns JE. Trabeculectomy: preliminary report of a method. Am J Ophthalmol 1968;66:673-9.
8 Sanders R, MacEwan CJ, Haining WM. Trabeculectomy: effect of varying the surgical site. Eye 1993;7:440-3.

9 Vernon SA, Gorman C, Zambarakji HJ. Medium to long-term intraocular pressure control following small flap trabeculectomy (microtrabeculectomy) in relatively low risk eyes. Br J Ophthalmol 1998;82:1383-6.

10 Vernon SA, Zambarakji HJ, Potgieter F, et al. Topographic and keratometric astigmatism up to 1 year following small flap trabeculectomy (microtrabeculectomy). Br J Ophthalmol 1999;83:779-82.

11 Zaidi AA. Trabeculectomy: a review and 4-year follow up. Br J Ophthalmol 1980;64:436-9.

12 Konstas AGP, Jay JL. Modification of trabeculectomy to avoid postoperative hyphaema: the "guarded anterior fistula operation." Br J Ophthalmol 1992;76:353-7.

13 Budenz DL, Hoffman K, Zacchei A. Glaucoma filtering bleb dysaesthesia. Am J Ophthalmol 2001;131:626-30.

14 Chauvaud D, Caly-fressinet C, Pouliquen Y, et al. Opacification of the crystalline lens after trabeculectomy. Arch Ophthalmol 1976;36:379-86.

15 AGIS Investigators. The advanced glaucoma intervention study. 8: Risk of cataract formation after trabeculectomy, Arch Ophthalmol 2001;119:1771-9.

16 The AGIS Investigators. The advanced glaucoma intervention study (AGIS): 7. The relationship between control of intraocular pressure and visual field deterioration. Am J Ophthalmol 2000;130:429-40. 